\title{
NUMERICAL CALCULATION OF ANISOTROPIC ABSORPTION FACTORS FOR LAMELLAR TWO-PHASE STRUCTURES
}

\author{
Y. S. LIU and H. J. BUNGE \\ Department of Physical Metallurgy, Technical University of Clausthal, FRG
}

(Received November 19, 1989)

\begin{abstract}
$\mathrm{X}$-ray diffraction in a lamellar two-phase material is considered. If the lamellae thicknesses are small compared with the penetration depth of the X-rays then the absorption factor depends on the whole path of incident and reflected beam in both phases. Particularly, it depends on a rotation $\gamma$ about the diffraction vector $s$ additional to the orientation $(\alpha \beta)$ of this vector with respect to the sample. Numerical calculations of the absorption factor $A(\alpha \beta \gamma)$ have been carried out for different values of lamellae thicknesses $D^{1} D^{2}$, absorption coefficients $\mu^{1} \mu^{2}$ and lamellae orientations $\omega \rho$ with respect to the sample surface.
\end{abstract}

KEY WORDS Absorption factor, lamellar structures, orientation dependence.

\section{INTRODUCTION}

$\mathrm{X}$-ray diffraction in polycrystalline, polyphase materials is the basis on which a number of structure parameters are being obtained, e.g. the volume fractions of the constitutive phases, their respective textures as well as residual stresses and others. The determination of the volume fractions of the phases and of the textures is based on intensity measurements of the reflected beam taken in different sample directions. The intensity of the diffracted beam is proportional to the volume fraction of the phase in which it is being diffracted. It is also proportional to the pole density of the corresponding lattice plane of this same phase, i.e. the pole figure of the reflecting lattice plane of this phase. These are properties of the considered phase which are independent of the presence of other phases and of the distribution of the phases. The intensity of the reflected beam is, however, also modified by absorption which takes place in all phases (Bunge 1985, 1986a). This latter factor thus depends on the absorption coefficients of all phases present in the sample and on their respective distribution in space as is illustrated schematically in Figure 1 for a two-phase material. Two limiting cases can be easily considered. If the particles of the diffracting phase are big compared to the penetration depth, Figure 1a, the absorption only takes place in the same phase as diffraction. Then there is virtually no difference compared with respect to a single phase material. If the particles are very small, Figure 1b then the incident and the reflected beams have to pass through very many particles of all phases weighted according to their respective volume fractions. The appropriate absorption factor of the material is then an averaged one of all participating phases. This case has generally been assumed in the literature when 

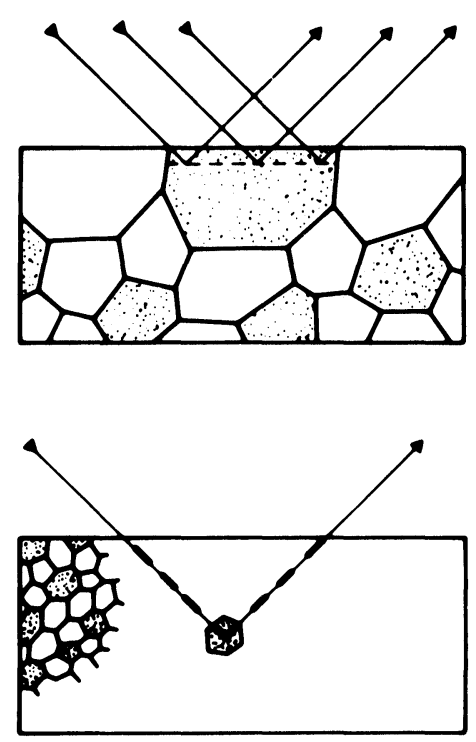

a

b

Figure 1 Diffraction in phase (1) of a two-phase material. (a) Big particles, (b) small particles.

multiphase diffraction is being considered (see e.g. Cullity, 1978). Naturally there must be a continuous transition from the big particle case to the small particle case. In an intermediate state the absorption factor may strongly depend on the sizes and the mutual arrangement of the particles of present phases. In this case the calculation of the effective absorption factor is extremely difficult. In fact, it is virtually impossible unless the real shapes and arrangements of the particles are known. There is, however, one intersting and practically important case of two-phase microstructure which can be sufficiently represented by a simple mathematical model, this is the case of lamellar structures as found in eutectics and eutectoids. In this case the structure consists of parallel lamellae with thicknesses $D^{1} D^{2}$ proportional to the volum fractions. This is a simple geometry for which the pathes of incident and reflected beam in both types of lamellae can be easily calculated. The partial beams reflected in different points of an individual lamella will have to pass through a varying number of lamellae of the same and all other phases depending on the angles of incident and reflected beam. It can not be expected that the absorption factor in this case can be expressed by a simple analytical formula. It can, however, be calculated numerically as was shown earlier for some specific cases (Bunge 1986, Hanneforth 1986, Bunge, Liu, Hanneforth 1987, Bunge, Hanneforth 1988).

It is the purpose of the present paper to describe the procedure of calculation for the most general case, i.e. lamellae of any sizes $D^{1} D^{2}$ with any absorption coefficients $\mu^{1} \mu^{2}$ and any orientation $(\omega \rho)$ with respect to the flat sample surface. Furthermore, Bragg reflection is considered with the Bragg-angle $\theta_{h k l}$ for any orientation of the diffraction vector $\mathbf{s}$ and for a rotation of the diffraction plane about the diffraction vector. The calculation program has been applied to a selection of values of the parameters. Since, however, the number of influencing parameters is so great, it is impossible to give a representative survey over all combinations of values of these parameters. Results of a large number of parameters can be found else where (Liu, Thesis, Clausthal). 


\section{FUNDAMENTALS OF CALCULATION}

We consider a lamellar two-phase structure as shown in Figure 2. The lamellae thicknesses are $D^{1}$ and $D^{2}$. The absorption coefficients of the two materials are $\mu^{1}$ and $\mu^{2}$. The lamellae are not perpendicular to the sample surface. The angle between the lamellae plane and the surface is $\omega$.

We chose a sample coordinate system $K_{A}=\left\{x_{1} x_{2} x_{3}\right\}$ in such a way that $x_{3}$ is the normal direction of the sample surface, $x_{2}$ is the direction of the trace of the lamellae in the sample surface and $x_{1}$ is perpendicular to the trace. The origin of $K_{A}$ is in the phase boundary such that the positive $x_{1}$-direction points into phase (1).

We also chose a lamellar coordinate system $K_{L}=\left\{y_{1} y_{2} y_{3}\right\}$ with the same origin (in Figure 2 shifted into the next lamella). Here $y_{3}$ is perpendicular to the plane of the lamellae and $y_{2}$ is parallel to the trace of the lamellae. The transformation between the two coordinate systems is

$$
\begin{aligned}
& y_{1}=x_{1} \cos \omega-x_{3} \sin \omega \\
& y_{2}=x_{2} \\
& y_{3}=x_{1} \sin \omega+x_{3} \cos \omega
\end{aligned}
$$

The lamellae thicknesses in the sample surface $d^{1}$ and $d^{2}$ are related to the true thicknesses by

$$
d^{1}=D^{1} / \sin \omega, \quad d^{2}=D^{2} / \sin \omega
$$

$\mathrm{X}$-ray diffraction is assumed to take place in the phase (1) in the point $\mathbf{p}$ with coordinates $\mathbf{p}=\left\{x_{1} x_{2} x_{3}\right\}$. Since the direction $x_{2}$ is parallel to the trace of the lamellae in the sample plane, the path lengths of incident and reflected beam in the phases (1) and (2) are independent of $x_{2}$. Hence, it is sufficient to consider diffraction points $\mathbf{p}$ in the $x_{1}-x_{3}$-plane, only. Furthermore, it is sufficient to consider only diffraction points in the first lamella. The trace of the phase

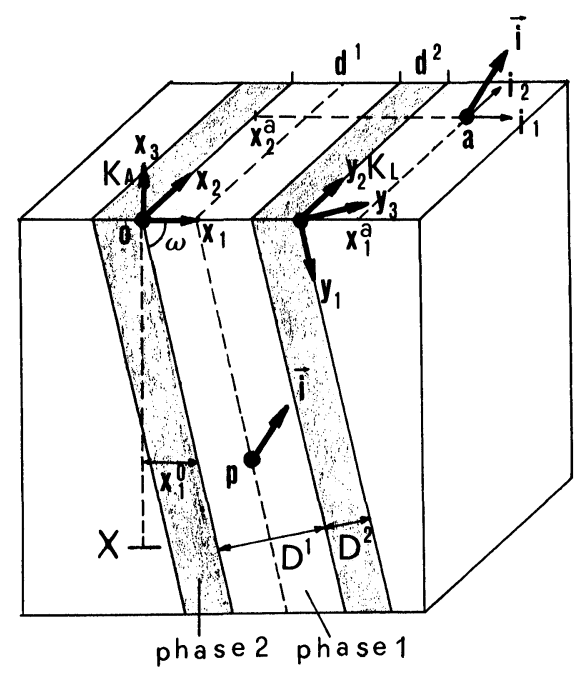

Figure 2 Definition of the quantities used in the calculation of the anisotropic absorption factor for a lamellar two-phase structure. 
boundary in this plane is given by

$$
x_{1}^{0}=-x_{3} \operatorname{ctg} \omega
$$

Hence, the range of diffraction points to be considered is

$$
\begin{aligned}
x_{1}^{0} & \leqslant x_{1} \leqslant x_{1}^{0}+d^{1} \\
0 & \leqslant x_{3} \leqslant-X
\end{aligned}
$$

We consider the incident and the reflected beam, both described by unit vectors $i$ and $\mathbf{r}$. These vectors will be described by their Cartesian coordinates in the systems $K_{A}$ and $K_{L}$ respectively as well as by their polar coordinates in the sample coordinate system $K_{A}$. It is for the incident beam

$$
\mathbf{i}=\left\{i_{1} i_{2} i_{3}\right\}_{K_{A}}=\left\{\phi_{i} \varphi_{i}\right\}_{K_{A}}=\left\{j_{1} j_{2} j_{3}\right\}_{K_{L}}
$$

The incident beam enters the sample through its surface. Hence, it is $i_{3}>0$. Furthermore, we shall need the relationship between Cartesian and polar coordinates

$$
\begin{aligned}
& i_{1}=\sin \phi \cos \varphi \\
& i_{2}=\sin \phi \sin \varphi \\
& i_{3}=\cos \phi
\end{aligned}
$$

And, according to Eq. (1), it is

$$
j_{3}=i_{1} \sin \omega+i_{3} \cos \omega
$$

The incident beam enters the sample (in the plane $x_{3}=0$ ) at the point a with the coordinates

$$
\mathbf{x}^{a}=\left\{x_{1}^{a} x_{2}^{a} 0\right\}
$$

which is related to the diffraction point $\mathbf{p}$ by

$$
\mathbf{x}^{a}=\mathbf{x}+L \cdot \mathbf{i}
$$

Hence, $L$ is the length of the path of the incident beam in the sample. With $x_{3}^{a}=0$ it follows from Eq. 9

and hence

$$
L=-\frac{x_{3}}{i_{3}}
$$

$$
x_{1}^{a}=x_{1}-x_{3} \frac{i_{1}}{i_{3}}
$$

The path of the incident beam in the two phases can be seen in Figure $3 b$ which represents the plane perpendicular to the sample surface containing the points $\mathbf{p}$ and $\mathbf{a}$ as is indicated in Figure 3a. It is assumed that the beam enters the sample in a lamella of phase (2), its path length in this lamella is $l_{0}^{2}$ and then it passes through $N$ more lamellae of this phase, the path length being $L^{2}$. The total path length in phase (2) is given by

$$
l^{2}=N \cdot L^{2}+l_{0}^{2}
$$

the path length $L^{2}$ is given by

$$
L^{2}=D^{2} /\left|j_{3}\right|
$$



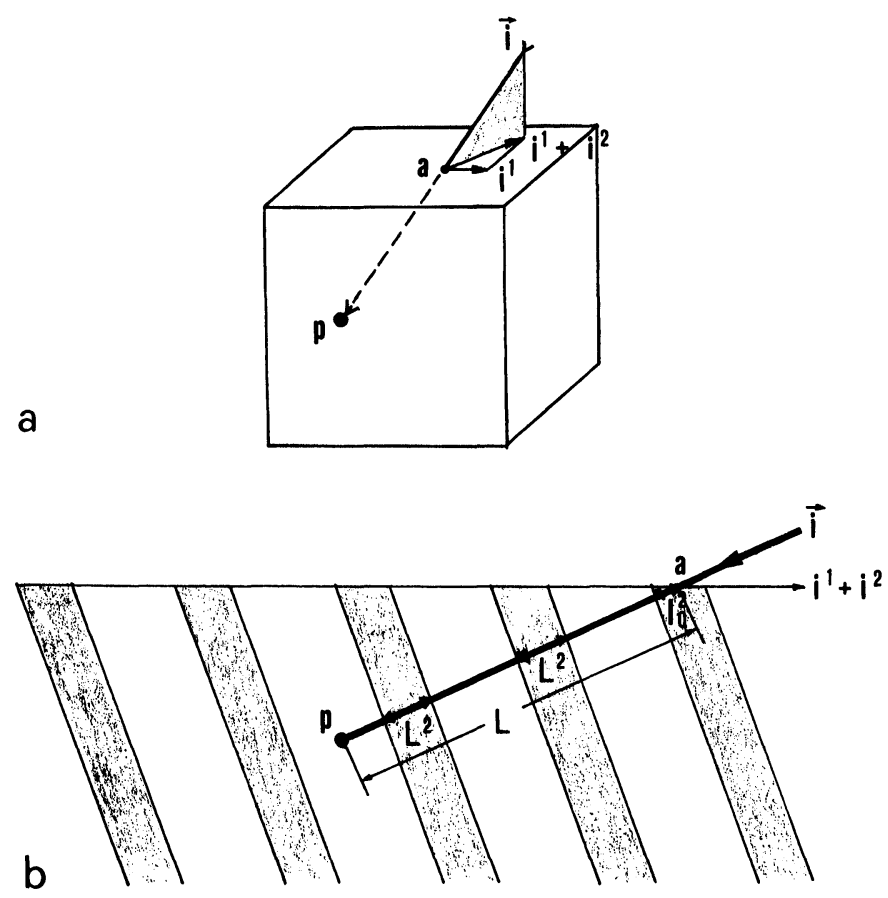

Figure 3 The incident beam in the direction of the unit vector $\mathbf{i}$ enters the sample at the point $\mathbf{a}$ and is reflected in point p. (a) Perspective representation of $\mathbf{i}$ with respect to the sample. (b) Section perpendicular to the sample surface containing the points $\mathbf{p}$ and $\mathbf{a}$.

If the beam enters the sample in phase (1) then it is $l_{0}^{2}=0$. The path length in phase (1) is given by

$$
l^{1}=L-l^{2}
$$

The number $N$ of phase (2) lamellae which are being passed completely can be obtained as is explained in Figure 4. Figure 4a shows the case that point $\mathbf{a}$ is in a phase (2) lamella whereas in Figure $4 \mathrm{~b}$ the point $\mathrm{a}$ is in a phase (1) lamella. The upper parts of the two figures are the $x_{3}=0$ projections, the lower ones the $x_{2}=0$ projections. Furthermore, the two cases $x_{1}^{a}>0$ and $x_{1}^{a}<0$ are shown.

At first we consider the distance $\delta$ in the upper part of Figure 4a. It is

$$
\begin{aligned}
& \delta=x_{1}^{a}-d^{1}-N\left(d^{1}+d^{2}\right) ; \quad x_{1}^{a}>0 \\
& \delta=-x_{1}^{a}-N\left(d^{1}+d^{2}\right) ; \quad x_{1}^{a}<0
\end{aligned}
$$

This distance is related to the path $l_{0}^{2}$ in the last lamella such that

$$
\delta=l_{0}^{2}\left[i_{1}+i_{3} \operatorname{ctg} \omega\right], \quad l_{0}^{2}=\frac{\delta}{i_{1}+i_{3} \operatorname{ctg} \omega}
$$

Next, we consider the distance $\delta_{n}$ of point a from the phase boundaries as 


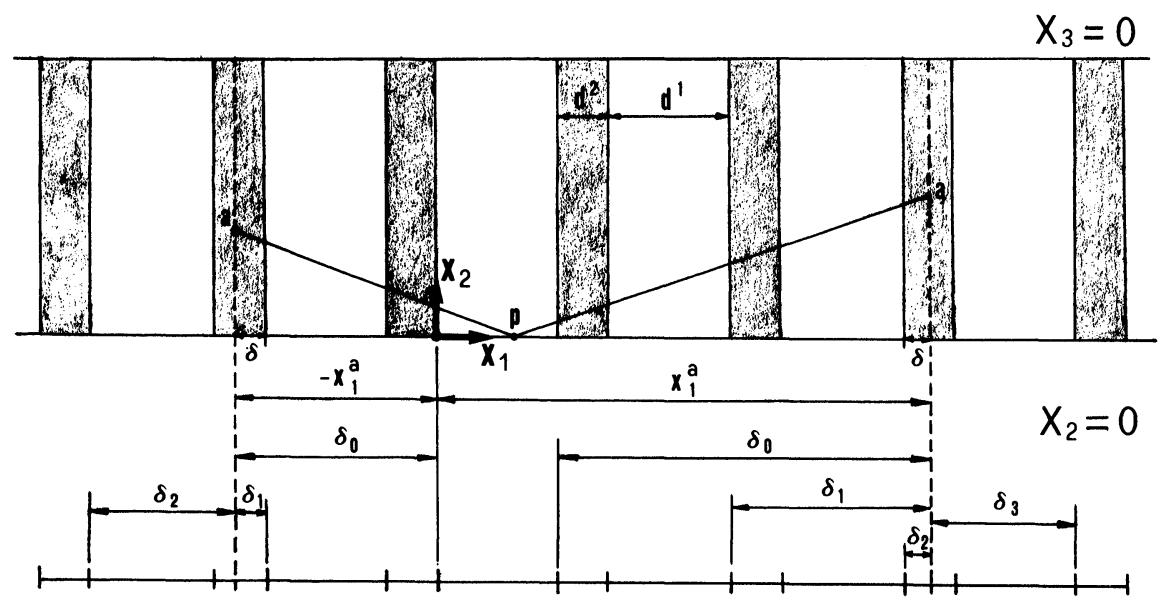

a

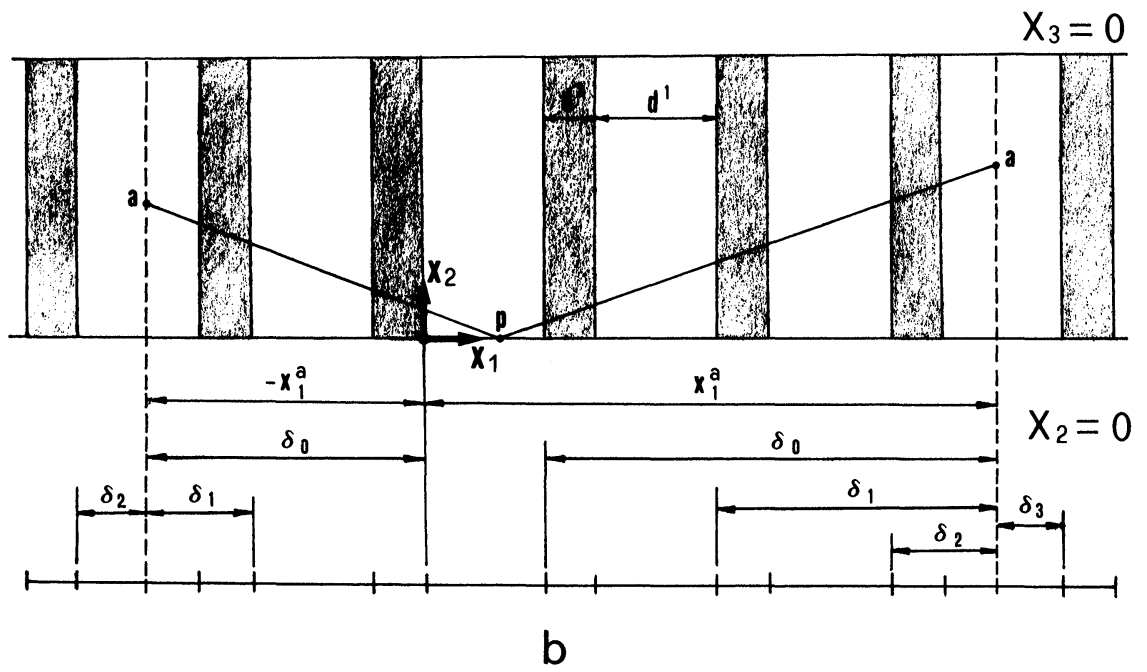

Figure 4 Definition of the quantities $\delta_{n}$. Upper parts: projection $x_{3}=0$ (sample surface). Lower parts: projection $x_{2}=0$ (sample front plane). (a) the incident beam enters the sample in a lamella of phase (2). (b) The incident beam enters the sample in a lamella of phase (1).

defined in Figure 4a, b lower part. It is

$$
\begin{array}{ll}
\delta_{n}=x_{1}^{a}-d^{1}-n\left(d^{1}+d^{2}\right) ; & x_{1}^{a}>0 \\
\delta_{n}=-x_{1}^{a}-n\left(d^{1}+d^{2}\right) ; & x_{1}^{a}<0
\end{array} \quad n=0,1,2, \ldots
$$

The number $N$ of entirely passed lamellae of phase (2) is defined such that

$$
\begin{array}{ll}
\delta_{n}>0 ; & \text { for } n=N \\
\delta_{n}<0 ; & \text { for } n=N+1
\end{array}
$$


Hence, one has to calculate the $\delta_{n}$ according to Eq. (17) and find the first negative one at the value $n=n^{-}$. Point a is in a phase (1) lamella if

$$
-\delta_{n^{-}}<d^{1}
$$

In this case we put

$$
N=n, \quad \delta=0
$$

Point $\mathbf{a}$ is in a phase (2) lamella if

$$
-\delta_{n^{-}}>d^{1}
$$

In this case we put

$$
N=n^{-}-1
$$

Hence, $N$ in Eq. (12) is known according to Eq. (20) or (22) and $l_{0}^{2}$ in Eq. (12) follows from Eq. (16) with $\delta$ according to Eq. (15) or Eq. (20). The path length of the incident beam in phase (1) and phase (2) is given by Eq. (12) and (14). Exactly the same considerations are to be made for the reflected beam. We put for the two beams

$$
\begin{array}{lll}
l^{1}=l_{i}^{1}, & l^{2}=l_{i}^{2} & \text { incident } \\
l^{1}=l_{r}^{1}, & l^{2}=l_{r}^{2} & \text { reflected }
\end{array}
$$

Then the total path lengths in the two phases are

$$
\begin{array}{ll}
\lambda^{1}=l_{i}^{1}+l_{r}^{1} & \text { phase (1) } \\
\lambda^{2}=l_{i}^{2}+l_{r}^{2} & \text { phase (2) }
\end{array}
$$

With these path lengths in the two phases we obtain the absorption factor

$$
\bar{A}=\frac{1}{X \cdot d^{1}} \int_{x_{3}=0}^{-X} \int_{x_{1}=x_{1}^{0}}^{x_{1}^{0}+d_{1}} e^{-\left[\mu^{1} \lambda^{1}+\mu^{2} \lambda^{2}\right]} d x_{1} d x_{3}
$$

Up to here the factor $\bar{A}$ was expressed in terms of the Cartesian components $\left\{i_{1} i_{2} i_{3}\right\}$ of the unit vector of the incident beam $\mathbf{i}$ and the corresponding components of the reflected beam $\mathbf{r}$.

These components were already expressed by the corresponding polar coordinates $\{\phi \varphi\}$ according to Eq. (6). It is, however, more convenient to express these coordinates by the polar coordinates $\{\alpha \beta\}$ of the diffraction vector $\mathbf{s}$, Figure 5 , the Bragg-angle $\theta$, and an additional angle $\gamma$ which fixes the orientation of the diffraction plane. The angle $\gamma=0$ is chosen such that the trace of the diffraction plane is perpendicular to the projection of the diffraction vector.

If the lamellae are polycrystalline and have a specific texture (including also the case of monocrystalline lamellae) then the intensity of diffraction is independent of the angle $\gamma$, a rotation about the diffraction vector. It depends only on the orientation $\{\alpha \beta\}$ of the diffraction vector. The angles $\alpha \beta$ are the normal pole figure angles which define the density of $(h k l)$-poles in the direction $\mathbf{s}$ of the diffraction vector. Hence, it is convenient to consider the absorption factor as a function of the Bragg angle $\theta$ and the three sample orientation angles $\{\alpha \beta \gamma\}$. The angles $\phi \varphi$ of incident and reflected beam can be expressed in terms of these 


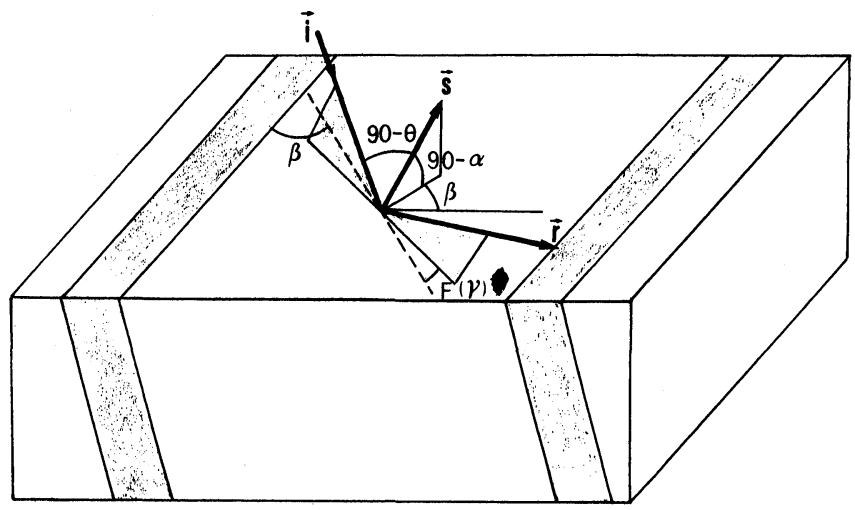

Figure 5 The diffraction plane containing incident $\mathbf{i}$ and reflected $\mathbf{r}$ beam and the diffraction vector $\mathbf{s}$. The latter one is fixed by its polar coordinates $\alpha \beta$. The diffraction plane is fixed by the rotation angle $\gamma$ about s. At $\gamma=0$ the trace of the diffraction plane is perpendicular to the projection of $\mathbf{s}$. The angle $F(\gamma)$ can be seen in Figure 6 .

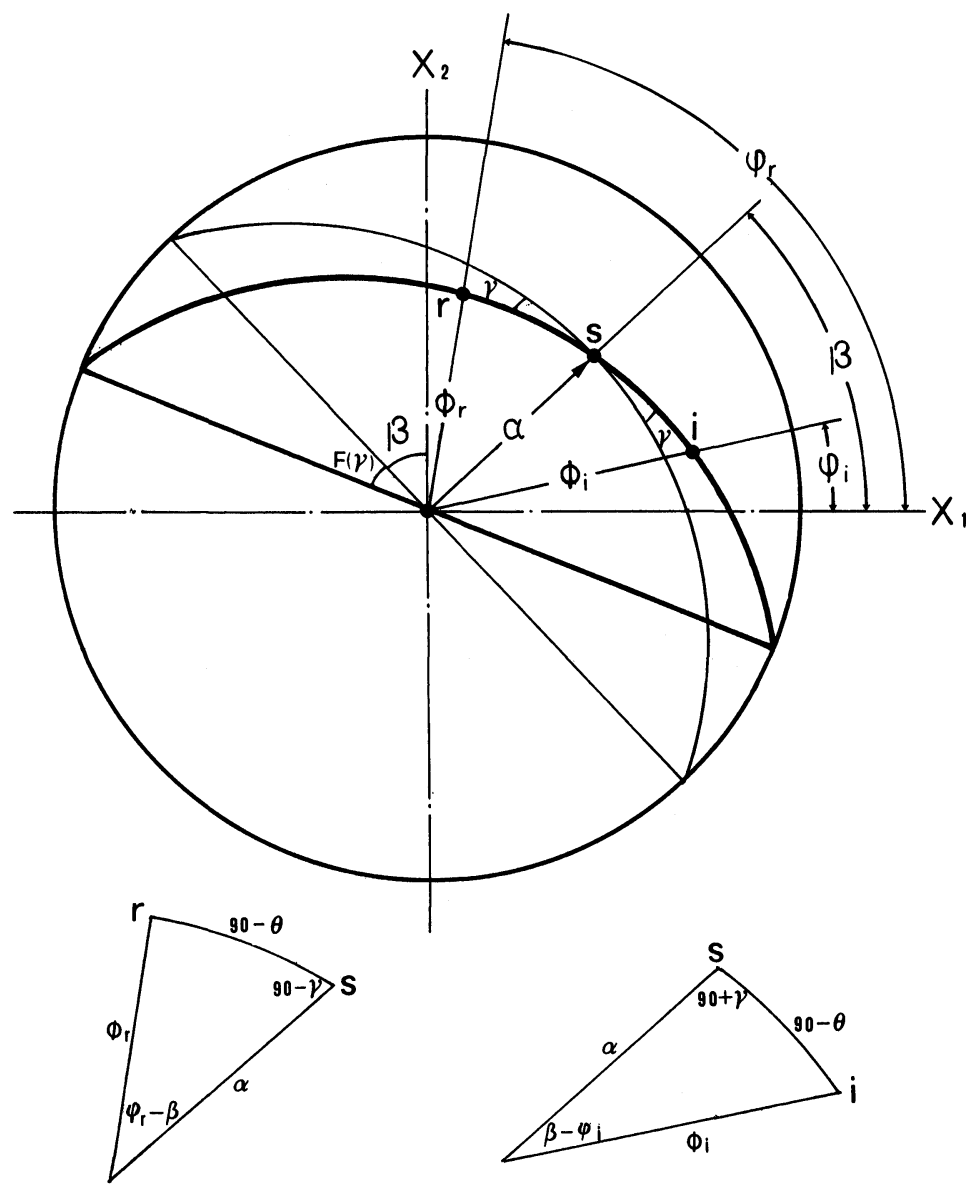

Figure 6 Relationship between the polar angles $\phi \varphi$ of incident and reflected beam and the sample orientation angles $\{\alpha \beta \gamma\}$ as defined in Figure 5 . 
angles. According to Figure 6 it is for the incident beam

$$
\begin{gathered}
\cos \phi^{i}=\cos \alpha \sin \theta-\sin \alpha \cos \theta \sin \gamma \\
\sin \left(\beta-\varphi^{i}\right)=\frac{\cos \gamma \cos \theta}{\sqrt{1-\cos ^{2} \phi^{i}}} \\
\cos \left(\beta-\varphi^{i}\right)=\frac{\sin \theta-\cos \phi^{i} \cos \alpha}{\sin \phi^{i} \sin \alpha}
\end{gathered}
$$

And for the reflected beam one obtains anologously

$$
\begin{gathered}
\cos \phi^{r}=\cos \alpha \sin \theta+\sin \alpha \cos \theta \sin \gamma \\
\sin \left(\beta-\varphi^{r}\right)=-\frac{\cos \gamma \cos \theta}{\sqrt{1-\cos ^{2} \phi^{r}}} \\
\cos \left(\beta-\varphi^{r}\right)=\frac{\sin \theta-\cos \phi^{r} \cos \alpha}{\sin \phi^{r} \sin \alpha}
\end{gathered}
$$

Both, incident and reflected beam must pass through the sample surface. Hence, it must be

$$
\begin{array}{ll}
\cos \phi>0 & \text { (incident) } \\
\text { (reflected) }
\end{array}
$$

With these relationships, the reflection factor $\bar{A}$ is expressed in terms of the variables

$$
\bar{A}=\bar{A}\left(D^{1}, D^{2}, \mu^{1}, \mu^{2}, \omega, \theta, \alpha, \beta, \gamma\right)
$$

Finally, for the most general case, we can omit the condition that the trace of the lamellae is parallel to $x_{2}$ (which was just the definition of the direction $x_{2}$ ). Hence, we rotate the sample coordinate system $K_{A}$ through the angle $\rho$ such that the trace of the lamella forms the angle $\rho$ with the $x_{2}$-direction, Figure 7 . This can be easily achieved by replacing $\beta$ in Eq. (33) with $\beta-\rho$. Hence, in the most general case, the absorption factor can be expressed in the form

$$
\bar{A}=\bar{A}\left(D^{1}, D^{2}, \mu^{1}, \mu^{2}, \omega, \theta, \alpha, \beta-\delta, \gamma\right)
$$

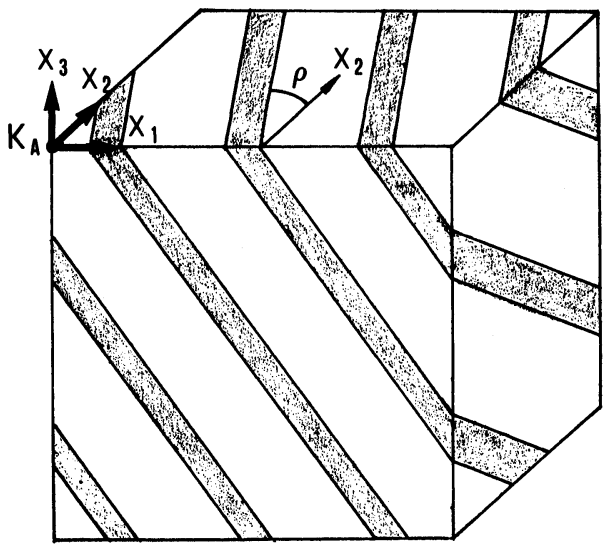

Figure 7 In the most general case the sample coordinate system $K_{A}$ is rotated through an angle $\rho$ with respect to the trace of the lamellae. 
For the purpose of n'merical calculation the integrals in Eq. (25) are to be replaced by finite sums We write

$$
\bar{A}=\frac{1}{M \cdot N} \sum_{m=0}^{M} \sum_{n=0}^{N} e^{-\left[\mu^{1} \lambda_{m n}^{1}+\mu^{2} \lambda_{m n}^{2}\right]}
$$

whereby the coordinates $x_{1} x_{3}$ of the diffraction point $\mathbf{p}$ which define $\lambda^{1}$ and $\lambda^{2}$ are expressed in terms of the step indices $m$ and $n$, Figure 8 .

$$
\begin{aligned}
x_{3}(m) & =\left(\frac{1}{2}+m\right) \Delta x_{3} \\
x_{1}(m, n) & =x_{1}^{0}(m)+\left(\frac{1}{2}+n\right) \Delta x_{1}
\end{aligned}
$$

and the steps are

$$
\begin{aligned}
& \Delta x_{3}=X / M \\
& \Delta x_{1}=d_{1} / N
\end{aligned}
$$

Appropriate values of $X, M, N$ have to be found empirically. They depend on the required accuracy.

In order to check numerical calculations it is useful to consider some limiting cases for which the absorption factor $\bar{A}$ can be calculated analytically. Generally, in a multiphase material the absorption factor is easily known for very big and very small phase particles as was shown in Figure 1. In these cases it is

$$
\begin{aligned}
A_{\text {big }} & =\frac{1}{\mu^{1}} \\
A_{\text {small }} & =\frac{1}{\bar{\mu}}=\frac{D^{1}+D^{2}}{\mu^{1} D^{1}+\mu^{2} D^{2}}
\end{aligned}
$$

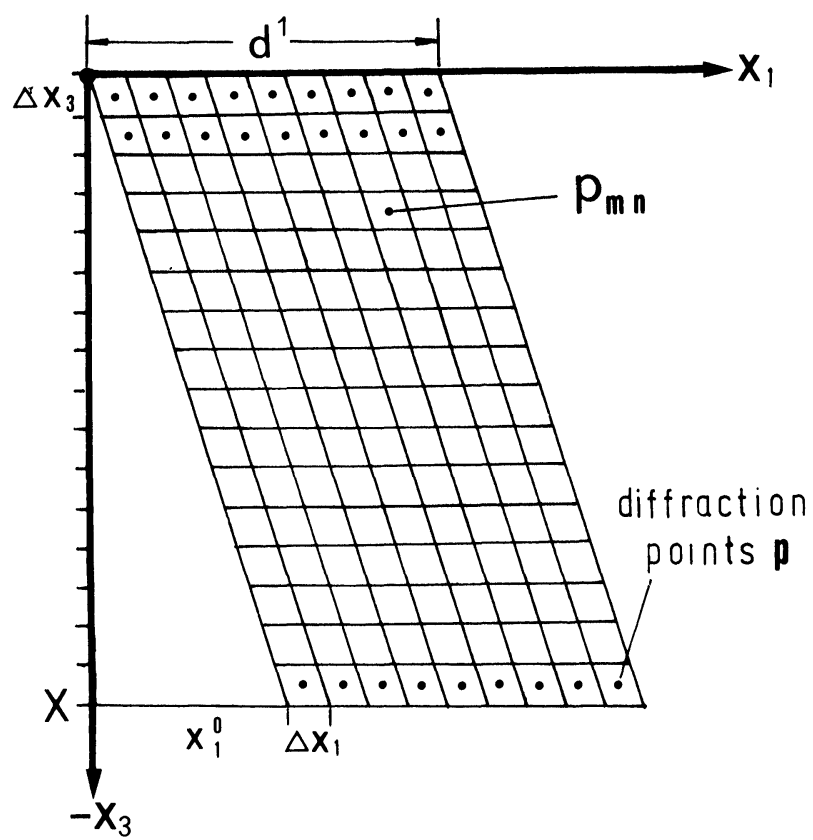

Figure 8 The integration interval is replaced by an equidistant net of summation points $m$ and $n$. 
Thereby $D^{1}$ and $D^{2}$ are the particle diameters in the directions of incident and reflected beam. In the case of lamellar structures with thin lamellae the "big particle" case applies if the diffraction plane is parallel to the lamellae plane and if it is at an angle, the "small particle" case holds, Figure 9. For thick lamellae there is no such distinction.

$$
\begin{array}{lll}
A_{\|}=A_{\text {big }}, & A_{\perp}=A_{\text {small }} & \mu \cdot D \ll 1 \\
A_{\|}=A_{\perp}=A_{\text {big }} & & \mu \cdot D \gg 1
\end{array}
$$

Hence, a good check of numerical calculations can be obtained by plotting $A_{\|} / A_{\perp}$

a
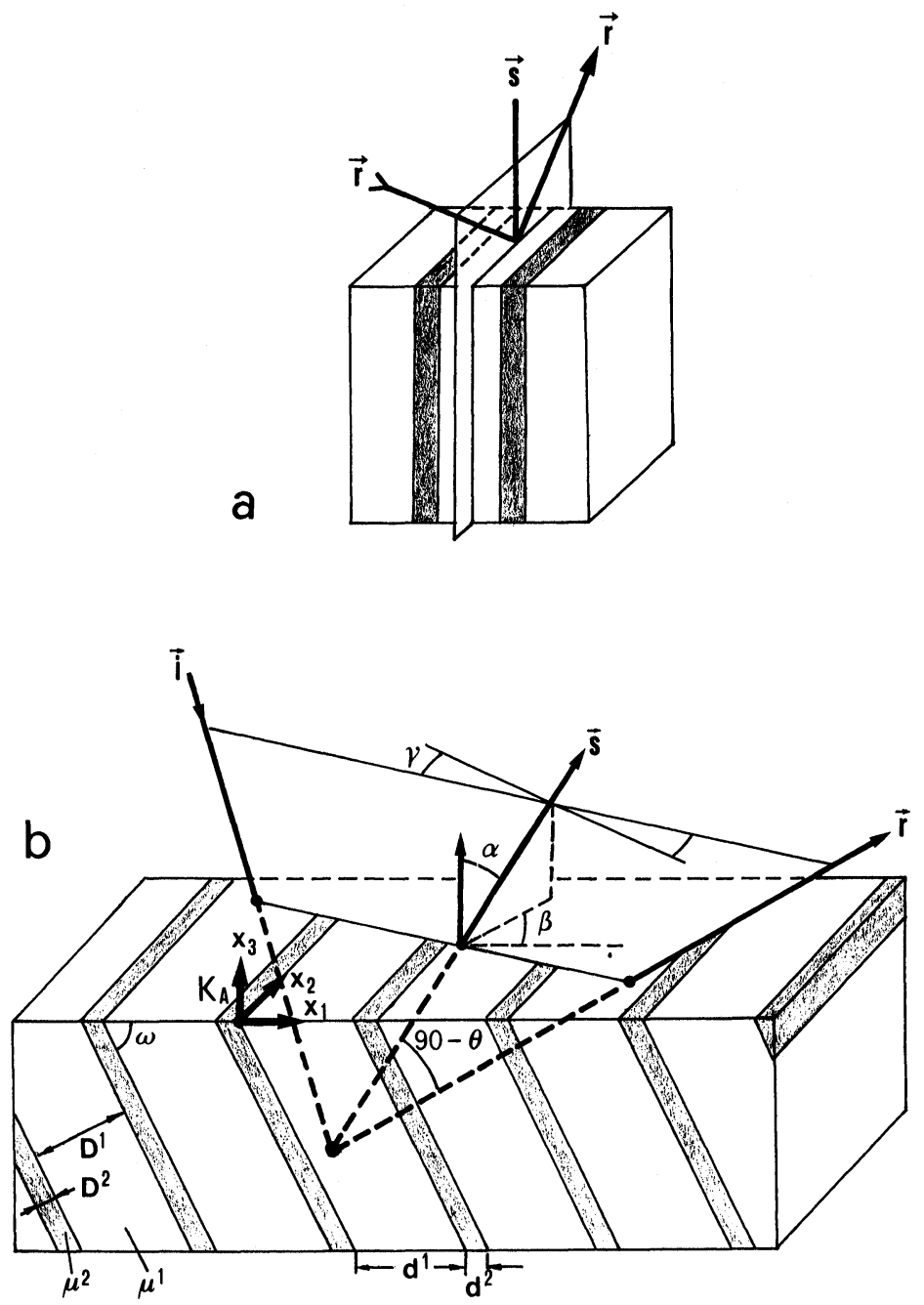

Figure 9 Diffraction of X-rays in a lamellar structure. (a) With the diffraction plane parallel to the plane of lamellae, (b) with the diffraction plane at an angle to the plane of lamellae. 
versus $\mu^{1} \cdot D^{1}$. The limiting case for "small particles" of this ratio is

$$
\frac{A_{\|}}{A_{\perp}}=\frac{D^{1} \mu^{1}+D^{2} \mu^{2}}{\left(D^{1}+D^{2}\right) \mu^{1}}=\frac{\frac{D^{1}}{D^{2}}+\frac{\mu^{2}}{\mu^{1}}}{\frac{D^{1}}{D^{2}}+1}
$$

\section{NUMERICAL CALCULATIONS}

In order to calculate the absorption factor $\bar{A}$ according to Eq. (35) depending on the variables given in Eq. (33), a computer program was written in FORTRAN (see Liu in preparation). The first step was to test the numerical accuracy of the calculation when the integral Eq. (25) was replaced by a sum Eq. (35) with a finite number of steps $m$ and $n$. The check was carried out by calculating the limiting values of $A_{\|} / A_{\perp}$ according to Eq. (40). This quantity is plotted in Figure 10 as a function of $\mu^{1} \cdot D^{1}$ for a particular case of $D^{1} / D^{2}$ but with different values of $m$ and $n$. It is seen that for $m=400, n=5$ (when $D \leqslant 1 \mu \mathrm{m}$ ) the results were in good agreement with the limiting values obtained from Eq. (40). In other cases other values of $m$ and $n$ had to be chosen in order to obtain sufficient accuracy with a minimum of computer time.

In Figure 11 further such curves are shown for a selection of values of $D^{1} D^{2} \mu^{1} \mu^{2}$. It is seen that in the limiting cases $\mu^{1} \cdot D^{1} \gg 1$ (big particle case) and $\mu^{1} \cdot D^{1} \ll 1$ (small particle case) there is no further dependence of $A_{\|} / A_{\perp}$ on $\mu^{1} \cdot D^{1}$. The curves show, however, that the transition from one to the other limit takes place with different slope and with different half-maximum values and also

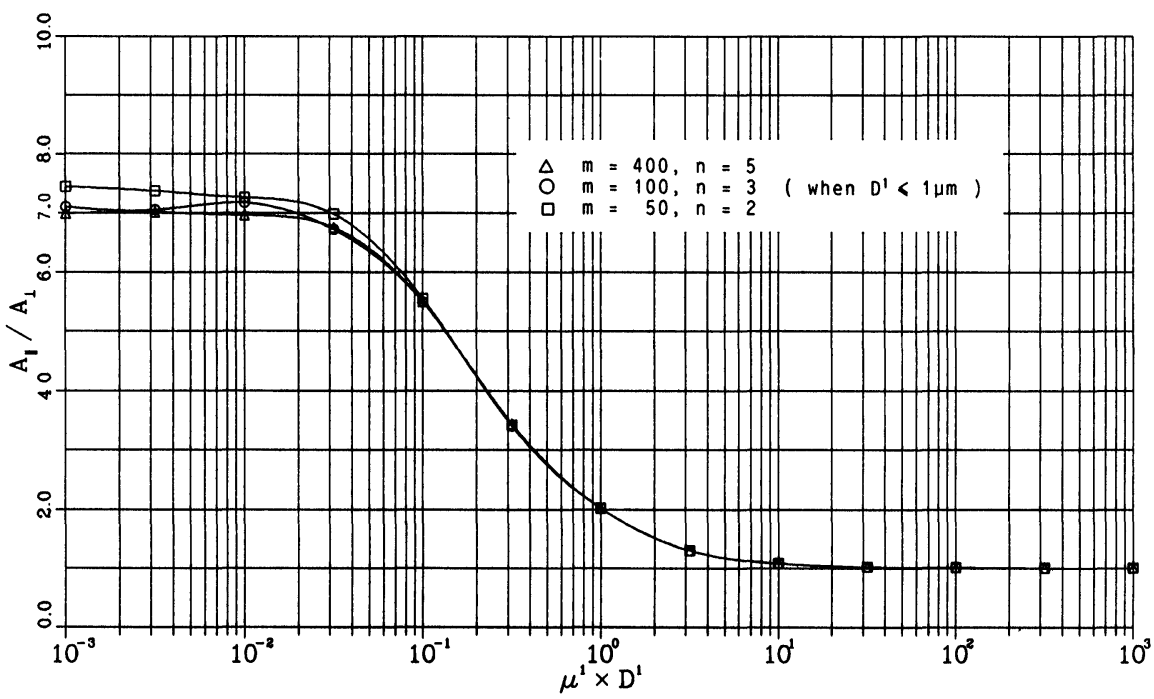

Figure 10 The quantity $A_{\|} / A_{\perp}$ as a function of $\mu^{1} \cdot D^{1}$ for $D^{1} / D^{2}=0.5, \mu^{1}=0.1 / \mu \mathrm{m}, \mu^{2}=1.0 / \mu \mathrm{m}$, $\omega=90^{\circ}, \theta=20^{\circ}$ for different numbers $m$ and $n$ of steps. 

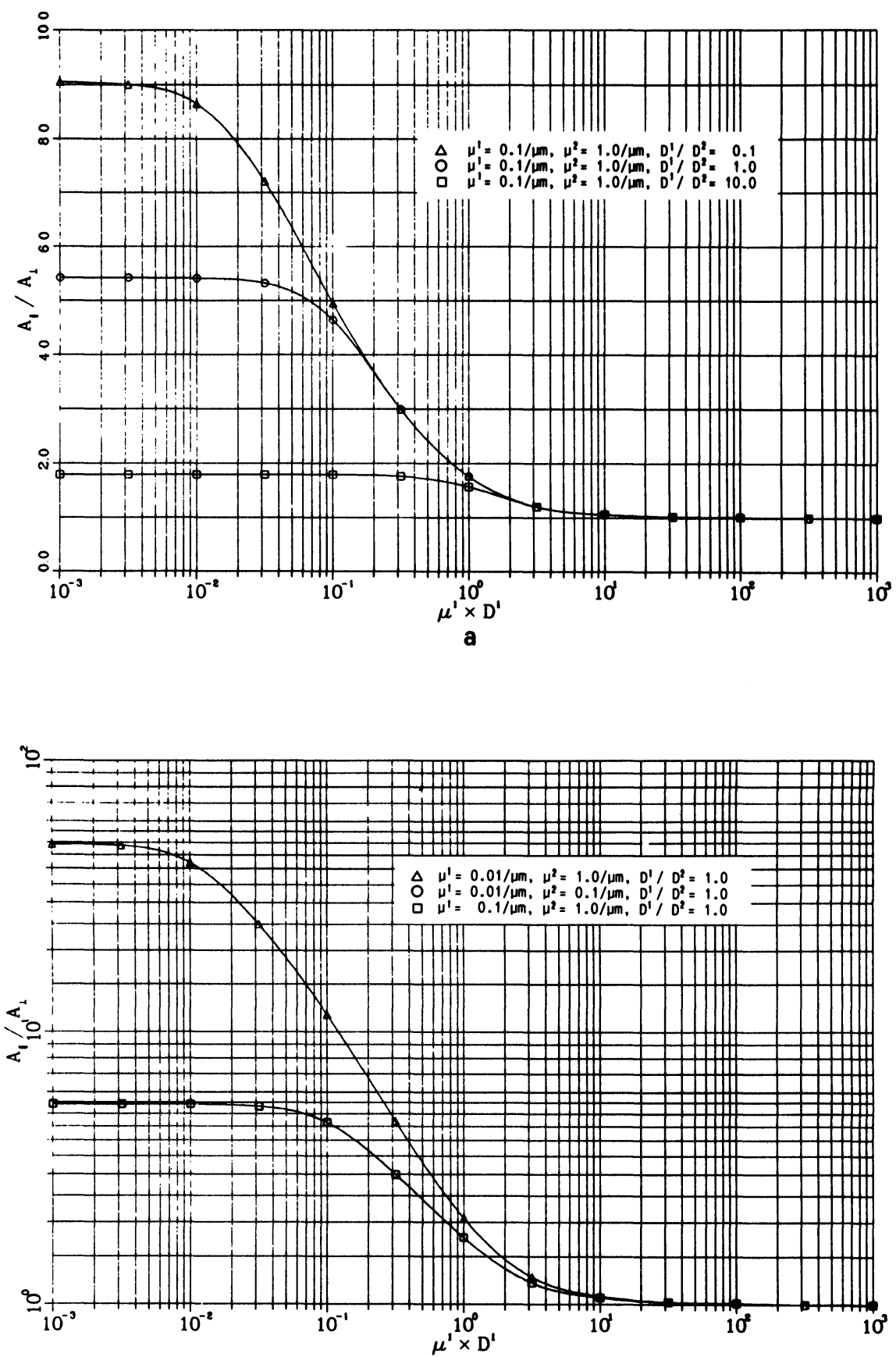

b

Figure 11. The quantity $A_{\|} / A_{\perp}$ as a function of $\mu^{1} \cdot D^{1}\left(\omega=90^{\circ}, \theta=45^{\circ}\right)$ for different values of $D^{1} / D^{2}(\mathrm{a})$ and different values of $\mu^{1}, \mu^{2}(\mathrm{~b})$. 

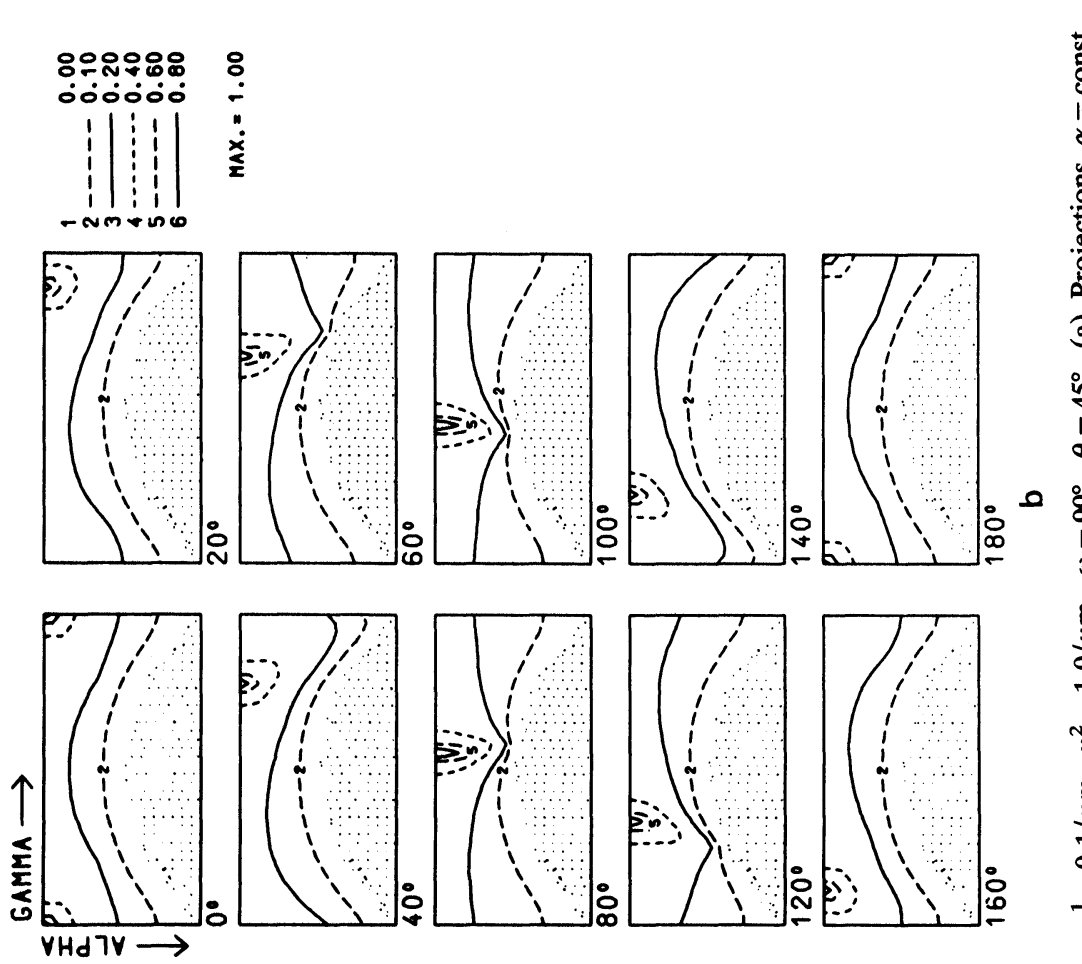

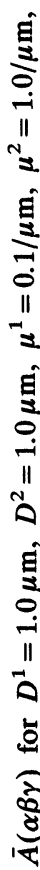
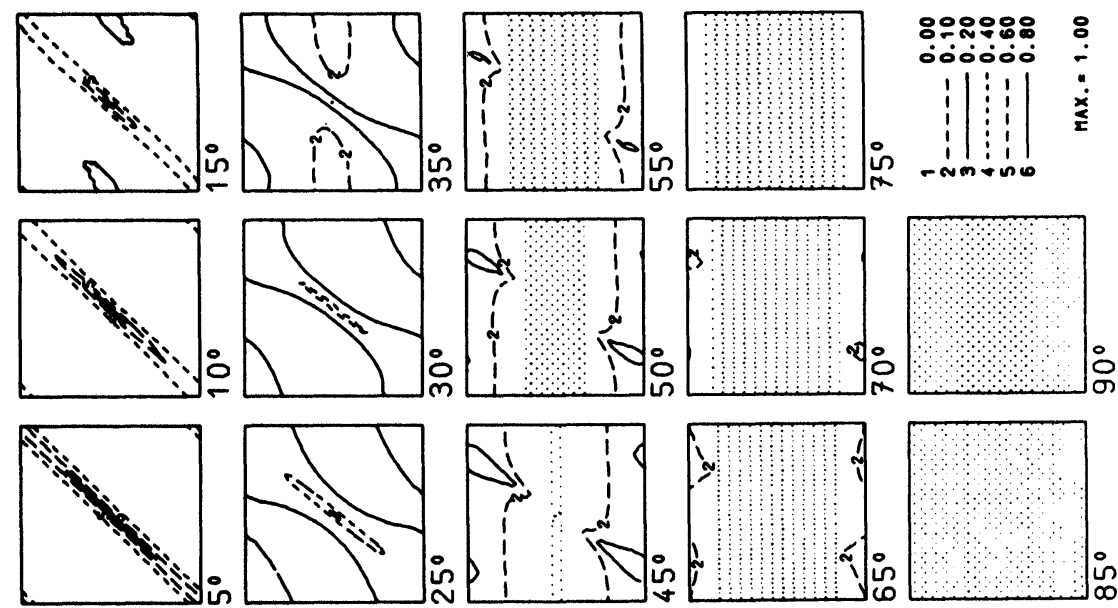

(o)
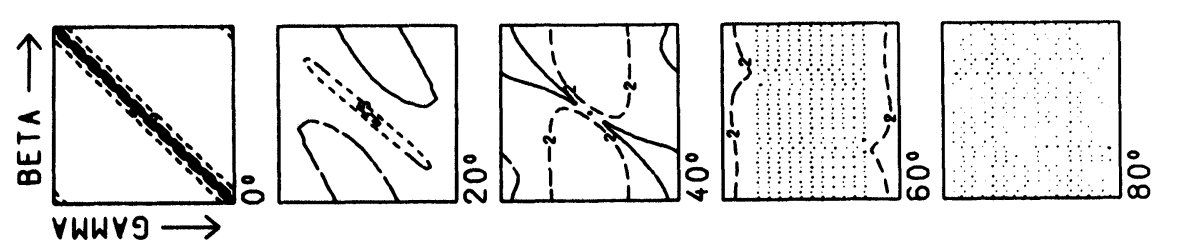

흉

ํㅜㄹ

ำํํำ

은 


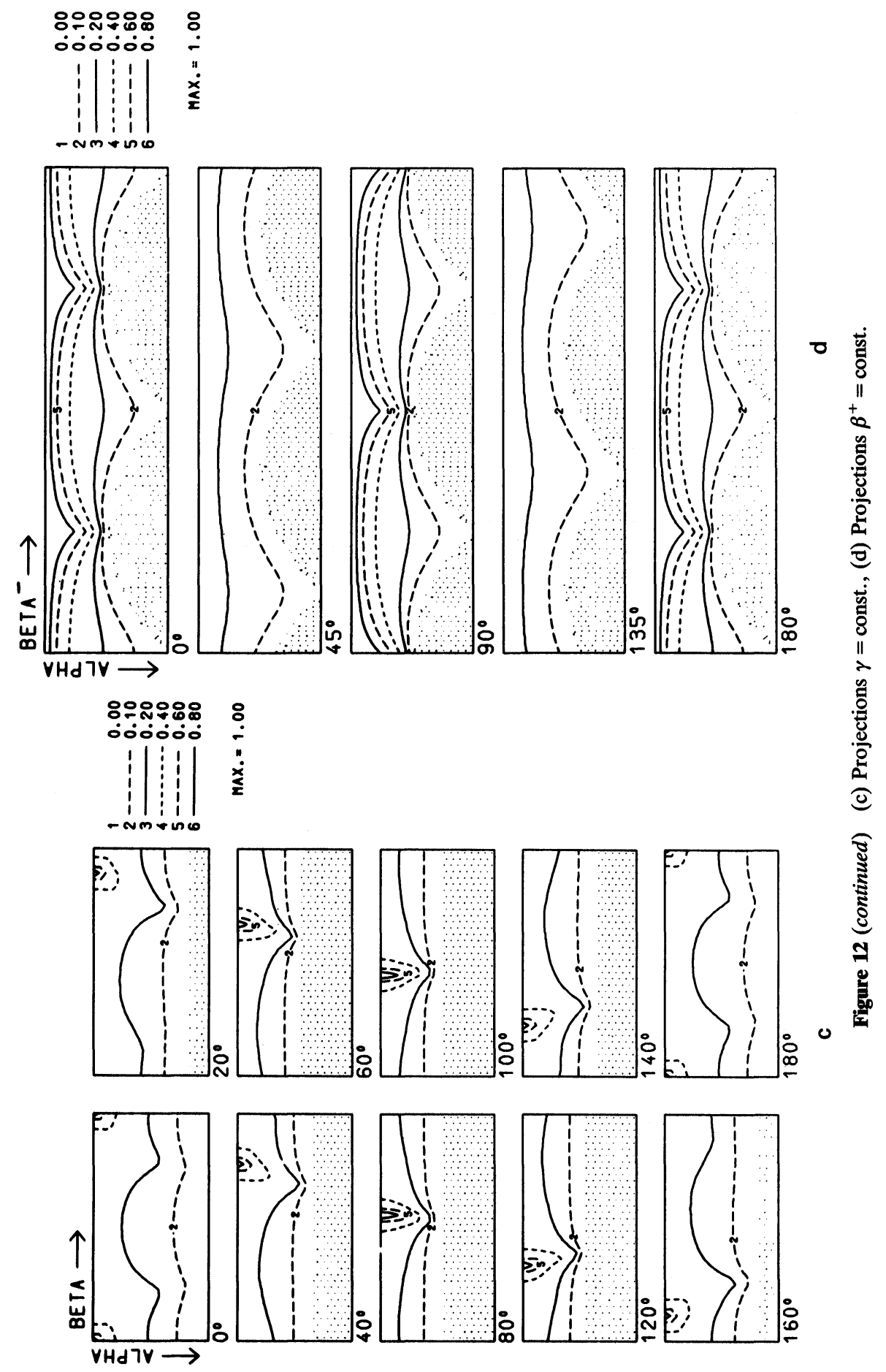



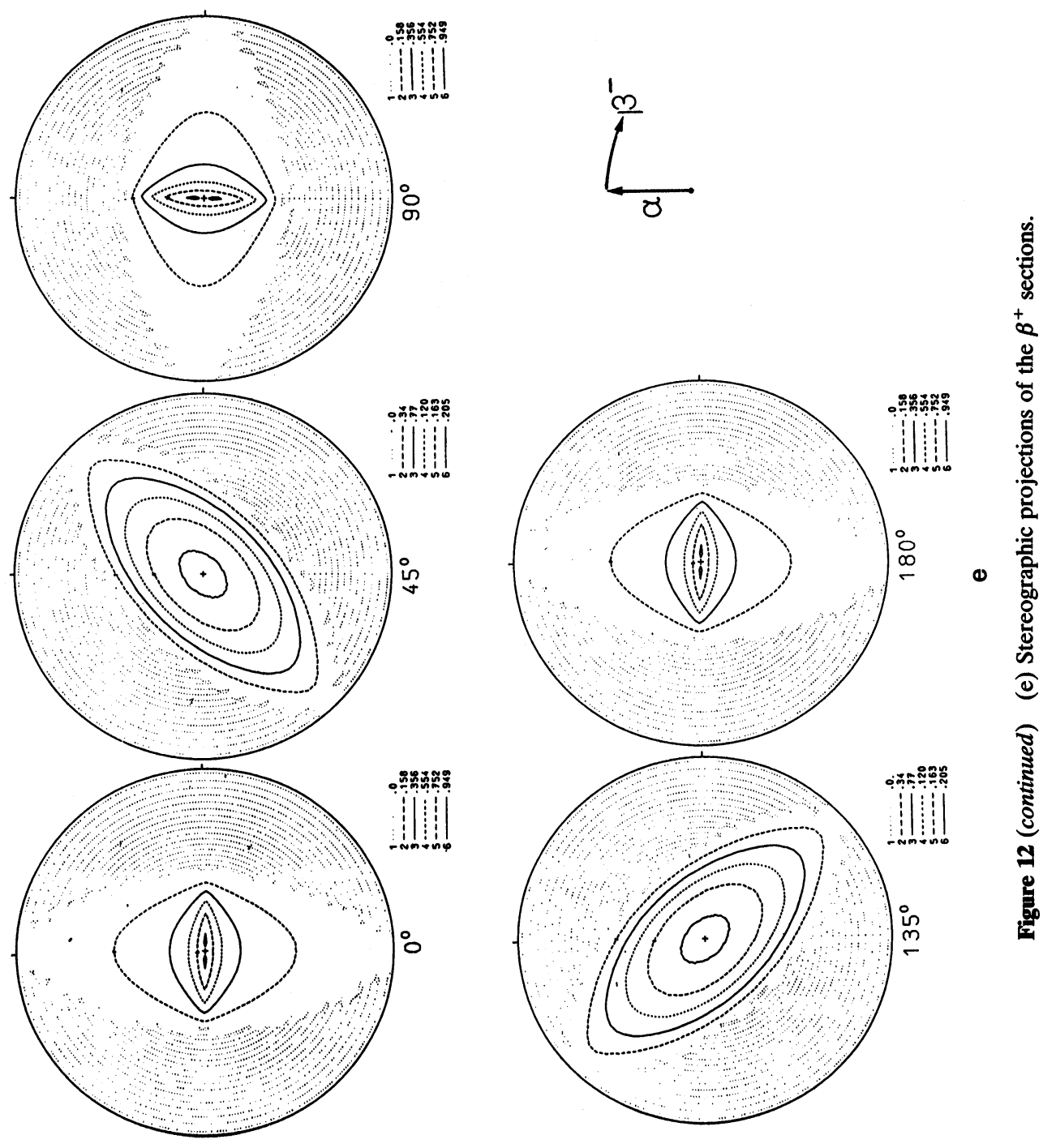
the limiting values themselves are strongly different. The curves of Figure 11 provide a good survey over the ranges of the variables $D^{1} D^{2} \mu^{1} \mu^{2}$ in which a strong anisotropic absorption effect occurs.

The variables in Eq. (33) may be divided into three groups. The first group $D^{1} D^{2} \mu^{1} \mu^{2} \omega$ characterize the sample whereas $\theta \alpha \beta \gamma$ describe the measurement process. These process variables in turn may be divided into the Bragg angle $\theta$ and the sample orientation angles $\alpha \beta \gamma$. In the conventional methods of texture measurement the Bragg angle is kept constant and the sample is rotated through two angles $\alpha \beta$ (the angular coordinates of the diffraction vector $s$ ). The result, the pole figure, is independent of the third sample angle $\gamma$. Hence it is reasonable to represent the absorption factor $\bar{A}$ as a function of the three orientation angles
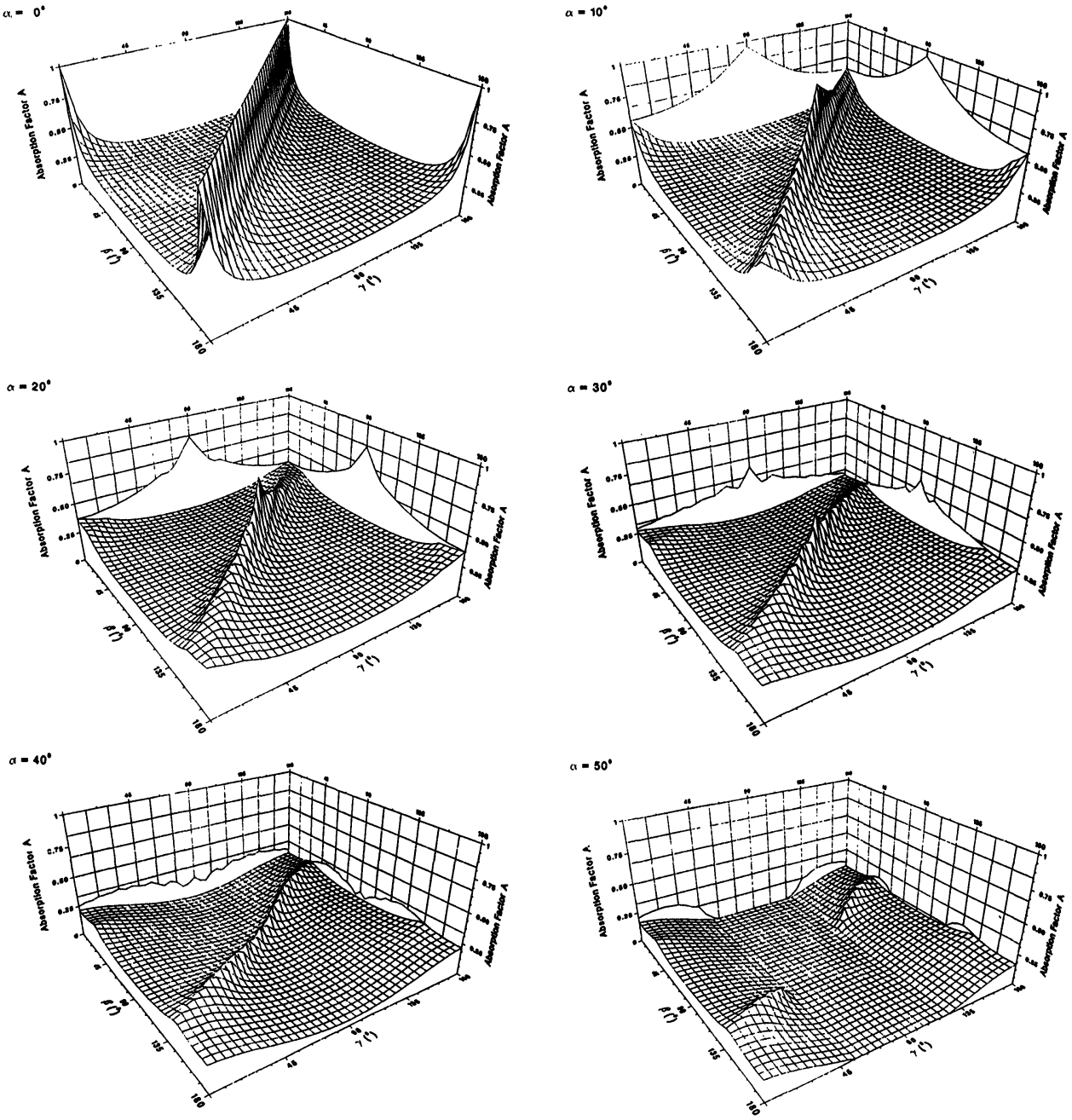

Figure 13 Perspective representation of $A(\alpha \beta \gamma)$ in $\alpha$-sections, $D^{1}=1.0 \mu \mathrm{m}, D^{2}=1.0 \mu \mathrm{m}, \mu^{1}=$ $0.1 / \mu \mathrm{m}, \mu^{2}=1.0 / \mu \mathrm{m}, \theta=45^{\circ}, \omega=90^{\circ}$. 
$\alpha \beta \gamma$ (Three-dimensional pole figures) for constant values of the other parameters $D^{1} D^{2} \mu^{1} \mu^{2} \omega$ and $\theta$.

There are many different ways how to represent a function of three variables. The situation is similar to the problem of how to represent the crystallographic orientation distribution function ODF (the texture). The angles $\alpha \beta \gamma$ characterize the sample orientation $\Gamma$ with respect to the coordinate system fixed in the $\mathrm{X}$-ray diffractometer. Figure 12 shows the results for a specific case in three different sections, i.e. sections for constant values of $\alpha, \beta, \gamma$ respectively. It is seen in Figure 12a that the $\alpha-\beta-\gamma$ space is degenerate at $\alpha=0^{\circ}$. Here the function depends only on $\beta+\gamma$. Hence, it seems reasonable to introduce new coordinates i.e.

$$
\begin{aligned}
& \beta^{+}=(\beta+\gamma) / 2 \\
& \beta^{-}=(\beta-\gamma) / 2
\end{aligned}
$$

similar to the representation of ODF in the coordinates $\varphi^{+}$and $\varphi^{-}$(Bunge, 1988). The $\beta^{+}$-representation of the same function is shown in Figure 12d. Finally the $\beta^{+}$sections are unique at $\alpha=0^{\circ}$. They can be represented in polar coordinates $\left\{\alpha \beta^{-}\right\}$and these in turn can be represented in stereographic projection. Hence, Figure 12e shows this representation.

A rather illustrative and translucent representation is the perspective representation of a two-dimensional field. In Figure 13 this representation is used for the $\alpha$-projections.

The most striking differences of the absorption factor occur if the diffraction vector $\mathbf{s}$ is in the lamellae plane. Figure $14 \mathrm{a}$ shows the absorption factor for $\alpha=\beta=0^{\circ}$ as a function of $\gamma$ for lamellae perpendicular to the sample surface $\omega=90^{\circ}$. Similar curves are given in Figure $14 \mathrm{~b}, \mathrm{c}$ for $\alpha=\omega-90^{\circ}, \beta=0^{\circ}$. With $\gamma=0^{\circ}$ the diffraction plane is parallel to the lamellae plane and with $\gamma=90^{\circ}$ it is perpendicular. All these curves are normalized such that

$$
A(000)=1
$$

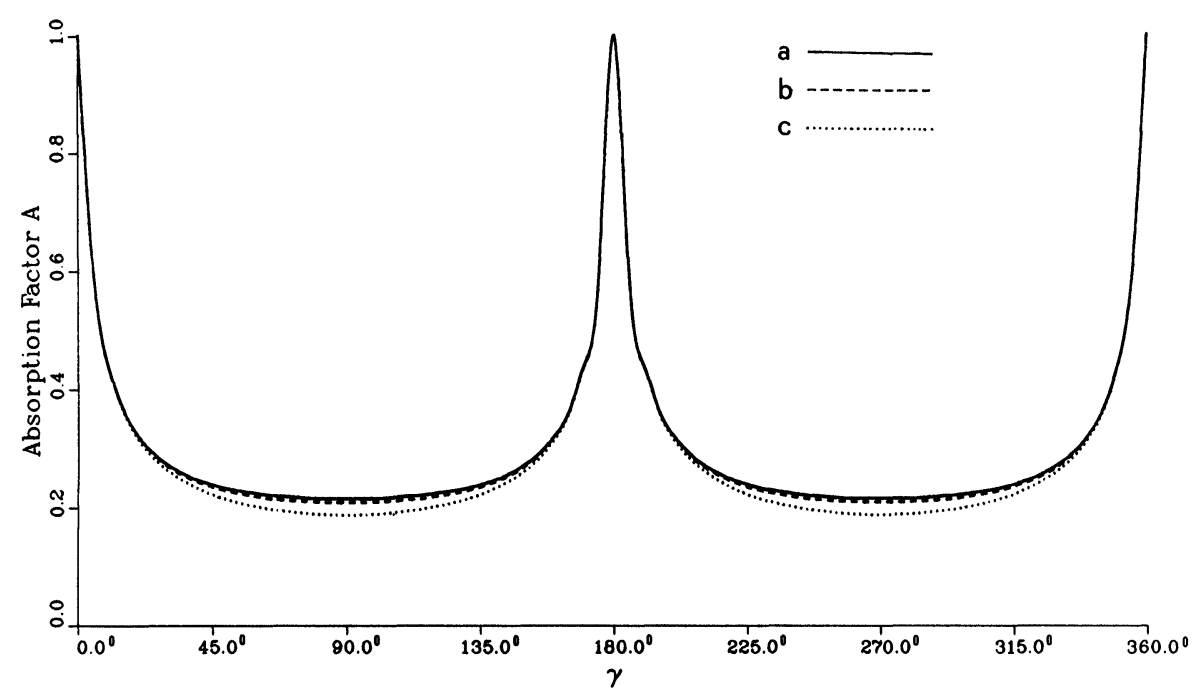

Figure 14 The absorption factor $A\left(\alpha=\omega-90^{\circ}, \beta=0^{\circ}, \gamma\right)$. (a) $\omega=90^{\circ}$, (b) $\omega=100^{\circ}$, (c) $\omega=110^{\circ}$. 


\section{References}

Bunge, H. J. (1985), Textures in Multiphase Alloys, Z. Metallkunde 76, 92-101.

Bunge, H. J. (1986a), Texture and Lamellae Distribution Function in Lamellar Eutectics, Textures and Microstructures 6, 265-287.

Bunge, H. J. (1986b), Anisotropic Absorption in Multiphase Texture Analysis, in: Experimental Techniques of Texture Analysis, Ed. H. J. Bunge, DGM Informationsgesellschaft, Oberursel, 395-402.

Bunge, H. J. (1988), Calculation and Representation of Complete ODF, in: Proc. ICOTOM 8, Eds. J. S. Kallend and G. Gottstein. The Metallurgical Society of AIME, 69-78.

Bunge, H. J. and Hanneforth, R. (1988), Calculation of Anisotropic Absorption Factors, in: Proc. ICOTOM 8, Eds. J. S. Kallend and G. Gottstein, The Metallurgical Society of AIME, 129-132.

Bunge, H. J., Liu, Y. S., Hanneforth, R. (1987), Anisotropic Absorption of X-rays in Polyphase Materials, Scripta Met., 21, 1423-1427.

Cullity, B. D. (1978), Elements of X-ray Diffraction, Addison-Wesley, Reading 409 pp.

Hanneforth, R. (1986), Berechnung anisotroper Absorptionsfaktoren für lamellare Verbundwerkstoffe, Diploma Work, Clausthal.

Liu, Y. S., Thesis, Clausthal (in preparation). 